\title{
Acquisition of Phrase-level Bilingual Correspondence using Dependency Structure
}

\author{
Kaoru Yamamoto and Yuji Matsumoto \\ Graduate School of Information Science, \\ Nara Institute of Science and Technology, \\ 8916-5, Takayama-cho, Ikoma-shi, Nara, Japan
}

\begin{abstract}
This paper describes a method to find phraselevel translation patterns from parallel corpora by applying dependency structure analysis. We use statistical dependency parsers to determine dependency relations between base phrases in a sentence. Our method is tested with a business expression corpus containing 10000 EnglishJapanese sentence pairs and achieved approximately $90 \%$ accuracy in extracting bilingual correspondences. The result, shows that the use of dependency relation helps to acquire interest-ing translation patterns.
\end{abstract}

\section{Introduction}

Since the advent of statistical methods in Machine Translation, the bilingual sentence alignment (Brown et al., 1991) or word aligmment (Dagan et al., 1992) have been explored and achieved numerous success over the last decade. In contrast, fewer results are reported in phraselevel correspondence. As word sequences are not translated literally a word for a word, acquiring phrase-level correspondence still remains an important problem to be exploited.

This paper proposes a method to extract phrase-level correspondence from sentence-aligned parallel corpora using statistically probable dependency relations, i.c. head-modifier relations in a sentence.

The distinct characteristics of our approach is two-fold. First, our approach uses dependency relations rather than alignment, cognate and/or position heuristics previously applied (Mclamed, 1995). Our approach is based on the assumption that the word ordering and positions may not necessarily coincide between the two languages, but the dependency structure between words will be preserved. We believe that dependency relations offer richer linguistic clues (syntactic information) and are effective for language pairs with different word ordering constraints.

Sccondly, statistical dependency parsers are used to obtain candidate patterns. Previous methods mostly use rule-based parsers for preprocessing(Maisumoto et al., 1993), (Kitamura and Matsumoto, 1995). The progress in parsing techmology are noteworthy, and in particular, various stalistical dependency models have been proposed(Collins, 1997), , (Ratnaparkhi, 1997), (Charniak, 2000). It has an advantage over the rule-based counterpart in that it achicves wider coverage, does not need to care for consistency in rule writing, and is robust to domain changes. We conjecture that our approach improves coverage and robusiness by use of statistical dependency parsers.

In this paper, we aim to investigate the efficacy of statistically probable dependency struct, ure in finding phrase-level bilingual correspondence. Though our discussion will proceed for English-Japanese phrasal correspondence, the proposed approach is applicable to any pair of languages.

This paper is organised as follows: In the next section, we present the overview of our approach. In Sections 3 and 4, components are claborated in detail. In Section 5, experiment and results are given. In Section 6, we compare our approach with related works, and finally our findings are concluded in Section 7.

\section{Overview of Our Approach}

Our approach presupposes a sentence-aligned parallel corpora. The task is divided into two steps: a monolingual step in which candidate patterns are generated by use of dependency relations, and a bilingual step in which these candidate patterns from each language are paired 


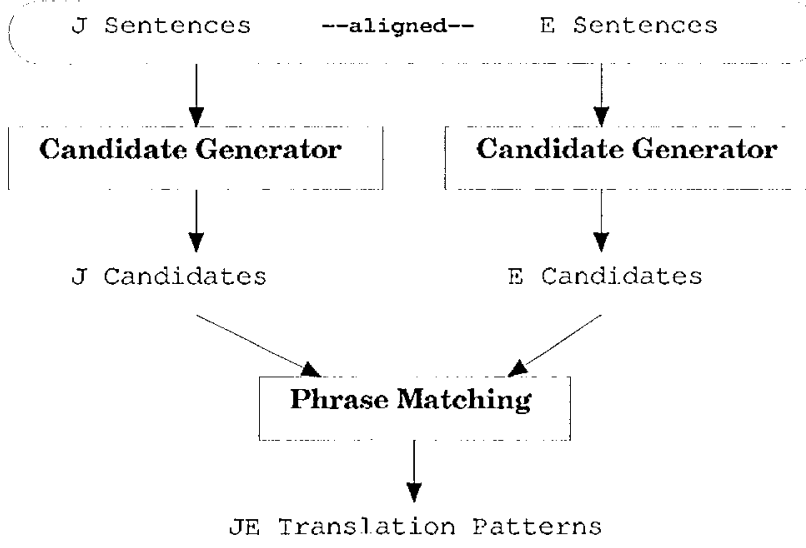

Figure 1: flow of our approach

with their translations. Figure1 shows the flow of our method.

Our primary aim is to investigate the effectiveness of dependency structures in the monolingual candidate generation step. For this reason, the bilingual step borrows the weighted Dice coefficient and greedy determination from (Kitamura and Matsumoto, 1996).

In the following sections, we cxplain each stcp in detail.

\section{Dependency-Preserving Candidate Patterns}

Dependency grammar or related paradigm (Hudson, 1984) focuses on individual words and their relationships. In this framework, every phrase is regarded as consisting of a governor and dependants, where dependants may be optionally classified further. The syntactically dominating word is solected as the governor, with modifiers and complements acting as dependants. Dependency structures are suitably depicted as a directed acyclic graph(DAG), where arrows direct from dependants to governors.

We use a maximum likelihood model proposed in (Fujio and Matsumoto, 1998) where the dependency probability between segments are determined based on its co-occurrence and distance. It has constraints that (a) dependencies do not cross, (b) each segment has at least one governor ${ }^{1}$. Furthermore, the model has an

\footnotetext{
${ }^{1}$ except for the 'root' segment. For Japanese, the 'root' segment is the rightmost segment. For English,
}

option to allow multiple dependencies whose probabilities are above certain confidence. It is useful for cases where phrasal dependencies cannot be determined correctly using only syntactic information. It has an effect of improving recall by sacrificing precision and may contain more partially correct results useful for our candidate pattern generation.

We apply the following notions as units of segments: For English, (a) a preposition or conjunction is grouped into the succeeding baseNPs ${ }^{2}$, (b) auxiliary verbs are grouped into the succeeding main vorb. For Japanese, one (or a sequence of) content word(s) optionally followed by function words ${ }^{3}$.

Having chunked into suilable segments, sentences are parsed to obtain dependency relations. We have setup the following three models:

1. best-one model : uses only the most likely (statistically best) dependency relations. At most one dependency is allowed for each segment.

2. ambiguous model : uses dependency relations above the certain confidence score $0.5^{4}$. Multiple dependencies may be considered for each segment.

3. adjacent model : uses only adjacency relations between segments. A segment is adjacent to the previous segment.

In the ambiguous model, we expect that more likely dependency relations will appear frequently given in a large corpus, thereby increasing the correlation score. Hence, ambiguity at parsing phase will hopefully resolved in the following bilingual pairing phase. As for the adjacent model, only chunking and its adjacency are used.

Finally, dependency relations between segments is used to generate candidate patterns.

the segment that contains the main verb is regarded as the 'root' segment.

2a baseNP or 'minimal' NP is non-recursive NP, i.e. none of its child constituents are NPs.

${ }^{3}$ often referred as a bunsetsu.

${ }^{4}$ statistically-not-the-best dependencies are also included if

$$
\frac{\operatorname{prob}(k t h-\text { ranked dependency })}{\operatorname{prob}((k+1) \text { th ranked dependency })} \geq 0.5
$$




\author{
[1] [saw] [a girl] [inl the park] \\ si.ze 1) $\{I$, saw, girl, park $\}$ \\ size 2) \{I_saw, girl_saw, in-park_saw\} \\ size 3) \{I_girl_saw(T), I in-park_saw(T)
}

Figure 2: best-one model

\author{
[I] [ǎa [agirl] [inl the park] \\ size 1) [I, sáw, girl, park\} \\ size 2) \{I_saw, girl_saw, in-park_saw, in-park_girl\} \\ size 3) \{I_girl_saw (T), I in-park_saw ( $(T)$, in-park girl_saw( $(\mathrm{L})\}$
}

Figure 3: ambiguous model

In this paper, dependency size of a candidate patitern designates the number of segments connected through dependency relations. Figures 2, 3, and 4 illustrate examples of Finglish candidate paticens of dependency size 1,2 and 3 for the proposed dependency models.

In a dependency-connected candidate pat-tern, function words of the governor segment is dropped. This is to cope with datia sparseness in generated candidate patierns. Moreover, two types of DAGs can be generated from patierns of size 3, and we use DAG-lype laggs (' $T$ ' and ' $\Gamma$ ') to distinguish their types. We also note 1.) hat candidate patierns do not necessarily follow the word ordering of original sentences.

The algorithm is as follows:

Input: a corpus, the minimum occurrence 1. Threshold in a corpus $f_{\min }$ and the dependency $\operatorname{size} d_{w}$.

For each sentence in a corpus, process the following:

1. Part-of-Specch Tagging

2. Chunking: Rules are written as regular cxpressions defined over POS word sequences.

3. Tependency Analysis

4. Candidate Pattern Generation: Candidate patterns are gencrated and stored with their sentence ID. Dependency-conmected patterns of less than or equal to the size $d_{w}$ are extracted.

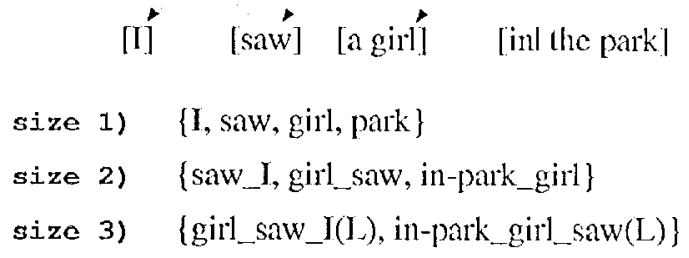

Figure 4: adjacent model

Output: a hash-table that maps from candidate patterns appearing at least the minimum occurrence $f_{\text {min }}$ to their sentence IDs found in the corpus.

\section{Phrase-level Correspondence Acquisition}

Pairing of candidate patterns is a combinatorial problem and we take the following tactics to reduce the search space. First, our algorithm works in a greedy manner. This means that a translation pair determined in the early stage of the algorithm will never be considered again.

Secondly, filtering process is incorporated. Figure 5 illustrates filtering for a sentence pair "I saw a girl in the park/私は公園の少女を見 tE". A set of candidate patterns derived from English is depicted on the left, while that from Japanese is depicted on the right. Once a pair “T_girl_saw(T)/私，少父を_見た ( termied as a translation pair, then the algo-

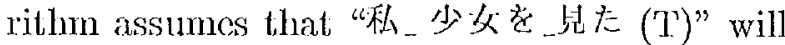
not be paired with candidate patterns related to "I_girl_saw(T)" (cancelled by diagonal lines in Figure 5) for the sentence pair. The opedation effectively discards the found pairs and causes recalculation of correlation scores in the procecding iterations.

As mentioned in Section 2, our corrclation score is calculated by the weighted Dice Coefficient defined as:

$$
\operatorname{sim}\left(p_{e}, p_{j}\right)=\left(\log _{2} f_{e j}\right) \frac{2 f_{e j}}{f_{e}+f_{j}}
$$

where $f_{j}$ and $f_{e}$ are the number of occurrences in Japanese and English corpora respectively and $\int_{e j}$ is the number of co-occurrences.

The algorilhm is as follows:

Input: hash-tables of candidate patterns for each language, the initial threshold of frequency $f_{\text {curr }}$ and the final threshold of frequency $f_{\text {min }}$. 


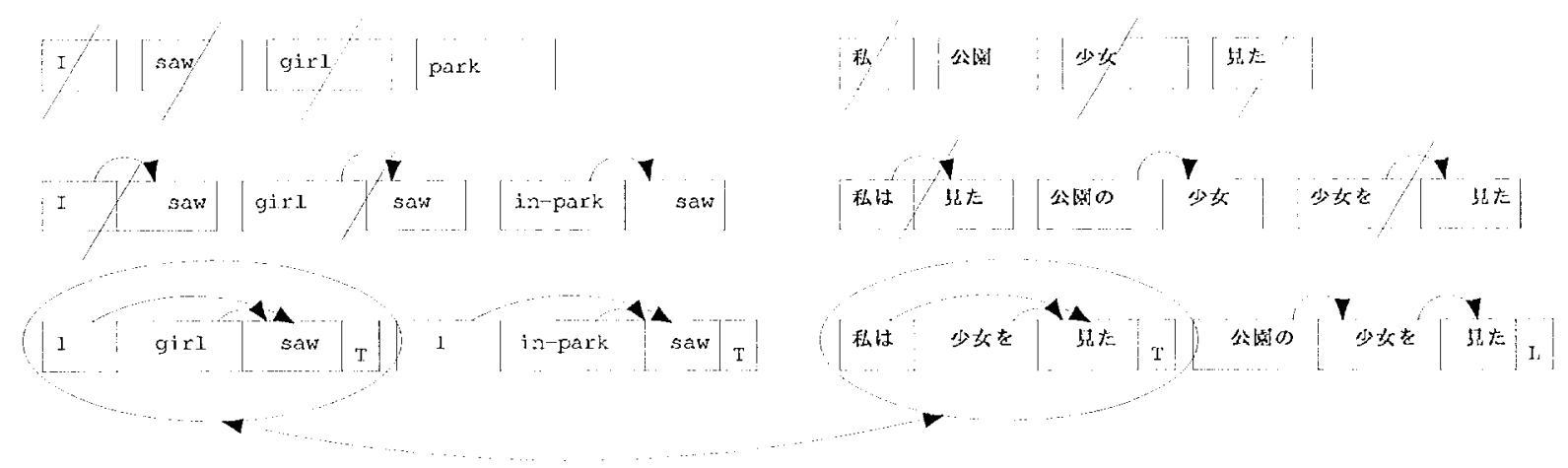

Figure 5: Filtering: word correspondence $=(\mathrm{I}$, 私 $)(\mathrm{saw}$, 見た $)($ girl, 少女 $)($ park, 公園 $)$

Repeat the following until $f_{c u r r}$ reaches $f_{\min }$.

1. For each pair of English candidate $p_{e}$ and Japanese candidate $p_{j}$ appearing at least $f_{\text {curr }}$ times, identify the most likely correspondences according to the correlation scores.

- For an English pattern $p_{e}$, obtain the correspondence candidate set PJ $=\{$ $\left.p_{j 1}, p_{j 2}, \ldots, p_{j n}\right\}$ such that $\operatorname{sim}\left(p_{e}, p_{j k}\right)$ $>\log _{2} f_{\min }$ for all k. Similarly, obtain the correspondence candidate set PE for an Japanese patiern $p_{j}$

- Register $\left(p_{e}, p_{j}\right)$ as a translation pair if $p_{j}=\operatorname{argmax} p_{j k} \in \mathrm{PJ} \operatorname{sim}\left(p_{e}, p_{j k}\right)$ and $p_{e}=\operatorname{argmax} p_{e k} \in \mathrm{PE} \operatorname{sim}\left(p_{j}\right.$, $\left.p_{e k}\right)$. The correlation score of $\left(p_{e}, p_{j}\right)$ is the highest among PJ for $p_{e}$ and PE for $p_{j}$.

2. Filter out the co-occurrence positions for $p_{e}, p_{j}$, and related candidate paticrns.

3. Lower the threshold of frequency if no more pairs are found with $f_{\text {curr }}$.

\section{Experiment and Result}

\subsection{Experimental Setting}

Wo use a business expression corpus (Takubo and Hashimoto, 1995) containing 10000 sentences pairs which are pre-aligned.

NLP tools are summarised in Table 1.

Parameter setting are as follows: dependency size $d_{w}$ is set to 3 . Initially, $f_{\text {curr }}$ and $f_{\min }$ are set to 100 and 2 respectively. As the algorithm proceeds, $f_{\text {curr }}$ is adjusted to half of its previous value if it is greater than 10 . Otherwise $f_{\text {curr }}$ is

\begin{tabular}{|l|l|l|}
\hline preprocessing & tool & \\
\hline POS(E) & ChaSen2.0 & $96 \%$ precision \\
POS(J) & ChaSen2.0 & $97 \%$ precision \\
\hline chunking(E) & SNPlex1.0 & rule-based \\
chunking(J) & Unit & rule-based \\
\hline dependency $(\mathrm{E})$ & edep & trial system \\
dependency $(J)$ & jdep & $85-87 \%$ precision \\
\hline
\end{tabular}

Table 1: NLP tools

decremented by 1 . If the number of registered translation pairs is less than 10 , then $f_{\text {curr }}$ is lowered in the next iteration. All parameters are empirically chosen.

\subsection{Result}

Our approach is evaluated by the metrics defined below:

$$
\begin{gathered}
\text { precision }=\frac{\operatorname{coun} t\left(p_{t}\right)}{\operatorname{count}\left(p_{x}\right)} \\
\text { coverage }=\frac{\sum_{p_{t}}\left(\operatorname{leng} \operatorname{th}\left(p_{l}\right) * \operatorname{cofreq}\left(p_{l}\right)\right)}{\sum_{p_{l}} \operatorname{occur}\left(p_{l}\right)}
\end{gathered}
$$

Precision measures the correctness of extracted translation pairs, while coverage measures the proportion of correct translation pairs in the parallel corpora. Let $X$ be a patitern. $\operatorname{count}(X)$ gives the number of $X$ returned, $\operatorname{occur}(X)$ gives the number of occurrences of $X$ in each corpus, length $(X)$ gives the dependency size of $\mathrm{X}$ and $\operatorname{cofreq}(X)$ gives the number of cooccurrences in the parallel corpora.. $p_{x}$ means extracted patterns, and of which correct patterns are designated as $p_{t} . p_{l}$ means the candidate patterns generated from each side of parallel corpora. Coverage is calculated for English 


\begin{tabular}{|r|r|r|r|r|}
\hline th & correct & extracted & c / o & precision \\
\hline 25 & 6 & 6 & 100.00 & 100.00 \\
12 & 7 & 7 & 100.00 & 95.00 \\
10 & 6 & 7 & 85.71 & 95.83 \\
9 & 4 & 4 & 100.00 & 92.30 \\
8 & 13 & 13 & 100.00 & 97.29 \\
7 & 10 & 13 & 76.92 & 92.00 \\
6 & 19 & 20 & 95.00 & 92.85 \\
5 & 29 & 29 & 100.00 & 94.94 \\
4 & 67 & 72 & 93.05 & 91.15 \\
3 & 150 & 164 & 91.16 & 92.83 \\
2 & 414 & 461 & 89.80 & 91.08 \\
(*2 & 264 & $\mathbf{1 7 4}$ & 55.69 & $77.93)$ \\
\hline total & $\mathbf{7 2 5}$ & $\mathbf{7 9 6}$ & -- & $\mathbf{9 1 . 0 8}$ \\
(*tatal & 989 & 1269 & - & $77.93)$ \\
\hline
\end{tabular}

Table 2: Precision: best--one model

\begin{tabular}{|r|r|r|r|r|}
\hline th & correct & extracted & $\mathrm{c} / \mathrm{o}$ & precision \\
\hline 25 & 6 & 6 & 100.00 & 100.00 \\
12 & 7 & 7 & 100.00 & 100.00 \\
10 & 6 & 7 & 85.71 & 95.00 \\
9 & 4 & 1 & 100.00 & 95.83 \\
8 & 13 & 13 & 100.00 & 97.29 \\
7 & 11 & 13 & 81.61 & 94.00 \\
6 & 18 & 19 & 94.73 & 94.20 \\
5 & 29 & 29 & 100.00 & 95.91 \\
4 & 68 & 73 & 93.15 & 94.73 \\
3 & 118 & 126 & 93.65 & 94.27 \\
2 & 132 & 168 & 91.50 & 93.07 \\
(*2 & 256 & 759 & 33.72 & $63.51)$ \\
\hline total & $\mathbf{7 1 2}$ & $\mathbf{7 6 5}$ & $-\cdot$ & $\mathbf{9 3 . 0 7}$ \\
(*tatal & 968 & 1521 & - & $\mathbf{6 3 . 5 1 )}$ \\
\hline
\end{tabular}

Table 3: Precision: ambiguous model

and Japanese separately and then thier mean is taken.

Precision for each model is summarised in Tables 2, 3, and 4, while coverage is shown in Table 5. To cxamine the characteristics of cach model, we expand correspondence candidate sets PE and PJ so that patterns ${ }^{5}$ with the correlation score $\geq \log _{2} 2(\geq 1)$ are also considered. These are marked by asterisks "*" in Tables.

Random samples of correct and near-correct translation pairs are shown in Table 6 , Table 7 respectively. Extracted translation pairs are matched against the original corpora to restore their word ordering. This restoration is done manually this time, but can be automated with little modification in our algorithm.

\footnotetext{
5i.c. pattcrns where $f_{e j}=f_{e}=f_{j}=f_{\text {min }}=2$
}

\begin{tabular}{|r|r|r|r|r|}
\hline th & correct & extracted & c / o & precision \\
\hline 25 & 6 & 6 & 100.00 & 100.00 \\
12 & 7 & 7 & 100.00 & 100.00 \\
10 & 6 & 7 & 85.71 & 95.00 \\
9 & 1 & 4 & 100.00 & 95.83 \\
8 & 13 & 13 & 100.00 & 97.29 \\
7 & 10 & 13 & 84.61 & 92.00 \\
6 & 18 & 19 & 94.73 & 92.75 \\
5 & 29 & 29 & 100.00 & 94.89 \\
4 & 68 & 73 & 93.15 & 94.15 \\
3 & 114 & 126 & 93.65 & 92.59 \\
2 & 119 & 484 & 86.57 & 88.86 \\
$\left({ }^{*} 2\right.$ & 280 & 496 & 56.45 & $76.27)$ \\
\hline total & $\mathbf{6 9 4}$ & $\mathbf{7 8 1}$ & - & 88.86 \\
*total & 974 & 1277 & - & $76.27)$ \\
\hline
\end{tabular}

Table 4: Precision: adjacent model

\begin{tabular}{|l|r|r|r|}
\hline model & English & Japanese & coverage \\
\hline best-one & $18.16 \%$ & $18.43 \%$ & $18.29 \%$ \\
best-one* & $19.12 \%$ & $19.59 \%$ & $19.13 \%$ \\
\hline ambiguous & $18.63 \%$ & $18.82 \%$ & $18.72 \%$ \\
ambiguous* & $19.57 \%$ & $19.95 \%$ & $19.76 \%$ \\
\hline adjacent & $17.74 \%$ & $18.03 \%$ & $17.88 \%$ \\
adjacent $^{*}$ & $18.69 \%$ & $19.20 \%$ & $18.94 \%$ \\
\hline
\end{tabular}

Table 5: Coverage

\subsection{Discussion}

As we see from Table 2 and 3, the best-one model achieves better precision than the adjacent model. Upon inspecting the results, nearly the same translation patierus are extracted for higher thresholds. This is because our dependency parsers use the distance feature in determining dependency. Consequently, nearer segments are likely to be dependency-related. Experiment data shows that the exact overlaps are found in 9348 out of $14705(63.55 \%)$ candidate patiterns for English and 6625 out of 11566 $(57.27 \%)$ for Japanese.

However, the difference appears when the threshold reaches 3 and patterns such as "not hesitate to contact/逶腎なくご連絡" which is not found in the adjacent model are extracted. Moreover, the best-one model is better in terms of coverage. These results supporti that the dependency relations appear useful clues than just being linearly ordered.

Comparing the best-one model with the ambiguous model, the ambiguous model achieves a higher precision except for $* 2$. T'his indicates 


\begin{tabular}{|c|c|c|}
\hline English & Japanese & score \\
\hline thank+you & ありがこう & 4.7037 \\
\hline tinclude & 協議_に_は十含める & 2.3219 \\
\hline he_position & 藪_に十応募_いたす & 2.2157 \\
\hline & て+お願い+中し上げる & 1.6000 \\
\hline & く+ご連絡 & 1.6000 \\
\hline & 封_いたす & 1.0566 \\
\hline be_v & もって十お知らせ_いたす & 1.0566 \\
\hline appli & こ』は十ある & 1.0000 \\
\hline E_direct & 十取締役_会 & 1.0000 \\
\hline & 中止_せ_ざる_を+得_なく+なる & 1.0000 \\
\hline$h_{\mathrm{r}}$ & 大いに十撕街_する & 1.0000 \\
\hline 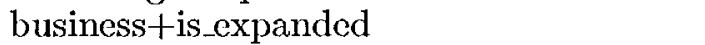 & 十発展_する & 1.0000 \\
\hline & 貴_ファックス_で十知る & 1.0000 \\
\hline$e^{\circ}$ & 約_1_0_日_後十出発 & 1.0000 \\
\hline elationship & 祭㣲_な十取引_四係_を十筑く & 1.0000 \\
\hline we+are & 权_W & 1.0000 \\
\hline pay+special_attentior & 特別_の十注澺_を十払う & 1.0000 \\
\hline
\end{tabular}

Table 6: random samples of correct translation patterns in best-one model. "+" indicates a scgmentseparator and "." indicates a morpheme-separator.

\begin{tabular}{|c|c|}
\hline English & Japanese \\
\hline $\begin{array}{l}\text { (have_been_pleased)+to_servet-as_thicr_main_banker } \\
\text { [be_held]+-at_hotel_new_ohtani } \\
\text { assets_position+(in_good_shape) } \\
\text { (have_becn_placed)+into_our_file } \\
\text { (put)+onc_month_limit } \\
\text { [passed]+on_past_tuesday }\end{array}$ & 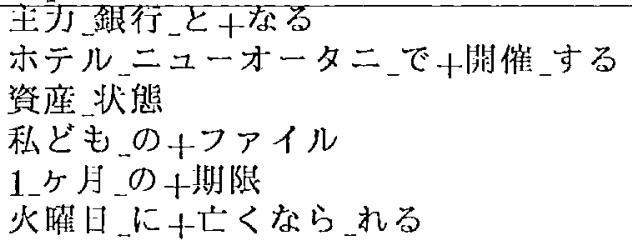 \\
\hline
\end{tabular}

Table 7: randam samples of near-correct translation patterns where score is 1.000 . Segments to be deleted to become correct patterns are embraced by "()". Segments to be added are cmbraced by "[]"

that, the accuracy of dependency parsers currently achieves are insufficient, and therefore, better to expand the possibilities of candidate patterns by allowing redundant dependency relations. As the dependency parsers improve, the best-one model will outperform the ambiguous model. However, as the result of $* 2$ shows, candidates from redundant dependency relations are mostly extractied at the low threshold. The overall trend reveals that redundant relations act as noise at low thresholds, but help to scale up the the correlation score at higher thresholds.

As shown in Table 6, a domain-specific disambiguation sample ("Thank you/ありがとう" vs. “Thank you in advance/前もってお願い申 し上げます”) is found. As for long-distance dependency-related translation patterns, "は"case (nominative) and verb patierns (consultations include/協誐には含める) are extracted ${ }^{6}$.

\footnotetext{
${ }^{6}$ A typical Japanese sentence follows S-O-V structure,
}

Other types of long-distance translation patterns such as "で"-case (accusative) and verb pallerns (be held al X/Xで開催する) are not extracled even candidate patterns from each corpus are generated.

Gencrally speaking, acquiring long-distance translation patterns is a hard problem. We still require further investigation examining under what circumstance the dependency relations are really cffective. So far, we use rclativcly "clcan" business expression corpora which is a collection of standard usage. However, in the real world setting, more repetitions and variations will be observed. Adjuncts can be placed in less constrained way and the adjacent model cannot deal with if they are apart. In such cases, availablilty of robusi dependency parsers become essential, dependency relations plays a key role in finding the long-distance translation patterns.

while the English counterpart follows S-V-O structure. 


\section{Related Works}

Smadja ct al.(1996) finds rigid and flexible collocations. They first identify candidate collocations in English, and subsequently, find the corresponding French collocations by gradually expanding the candidate word sequences. Kitamura et al.(1996) enumerates word sequences of arbitrary length (n-gram of content words) that appear more than the minimum threshold from English and Japanese and attempts to find the correspondence based on the prepared candidate lists.

Difference from Smadja et al.(1996) is that our method is bi-directional and difference from Kitamura et al. (1996) is that we use dependency relations which leads to "structured" phrasal correspondence as opposed to "flat:" adjacent correspondence.

On the other hand, Matsumoto et al.(1993), Kilamura et al.(1995) and Meyers et al.(1996) use dependency structure for structural matching of sentences to acquire translation rules. Their methods employ grammar-based parsers and only work for declarative sentences. Their objectives are complete matching of dependency trees of two languages.

Instead, our method uses statistical dependency parsers and are not restricted to simple sentences for input. Trurthermore, we are concerned with partial matching of dependency trees so that, the overall robustness and coverage will be improved.

\section{Conclusion}

In this paper, we propose a method to find phrase-level bilingual correspondence using dependency structure from parallel corpora. We have conducted a preliminary experiment with 10000 business sentence pairs of English and Japanese and achieved approximately $90 \%$ precision.

Though a fuller investigation still requires, our finding shows that the dependency relations serve as useful linguistic clues in the task of phrase-level bilingual correspondence acquisition.

\section{References}

P.JP. Brown, J.C. Lai, and R.I. Mercer. 1991. Aligning sentences in parallel corpora. In
ACL-29: 29th Annual Meeting of the Associalion for Compulational Linguistics, pages 169-176.

E. Charniak. 2000. A maximum-cntropyinspired parser. In NAACL-2000: 1st Meeting of the North American Chapter of the Association for Computational Linguistics, pages $132-139$.

M.J. Collins. 1997. Three generative, lexicalised models for statistical parsing. In ACL-97: 35th Annual Meeting of the Association for Compulational Linguistics, pages 16-23.

I. Dagan, K. Church, and W. Gale. 1992. Robust bilingual word alignment for machine aided translation. In Proc. of the Workshop on Very Large Corpora, pages 1-8.

M. Fujio and Y. Matsumoto. 1998. Japanese dependency structure analysis based on lexicalized statistics. In Proc. of $3 r d$ Conf. on Emperical Methods in Natural Language Processing, pages 88-96.

R. Hudson. 1984. Word Grammar. Blackwell.

M. Kitamura and Y. Matsumoto. 1995. A machine translation system based on translation rules acquired from parallel corpora. In Proc. of Recent Advances in Nalural Lannguage Processing, pages 27-44.

M. Kitamura and Y. Matsumoto. 1996. Automatic extraction of word sequence correspondences in parallel corpora. In Proc. 4th Workshop on Very Large Corpora, pages 7987.

Y. Matsumoto, H. Ishimoto, and T. Utsuro. 1993. Structural matching of parallel texts. In ACL-93: 31st Annual Mecting of the Association for Compulalional Linguistics, pages 23-30.

I.D. Melamed. 1995. Automatic evaluation and uniform filter cascades for inducing n-best translation lexicons. In Proc. of 3rd Workshop on Very Large Corpora, pages 184 198.

A. Ratnaparkhi. 1997. A lincar observed time stalistical parser based on maximum cntropy models. In Proc of 2nd Conf. on Emperical Methods in Natural Language Processing, pages 1-10.

K. Takubo and M. Hashimoto. 1995. A Dictionary of English Bussiness Letter Expressions. Nihon Keizai Shimbun, Inc. 